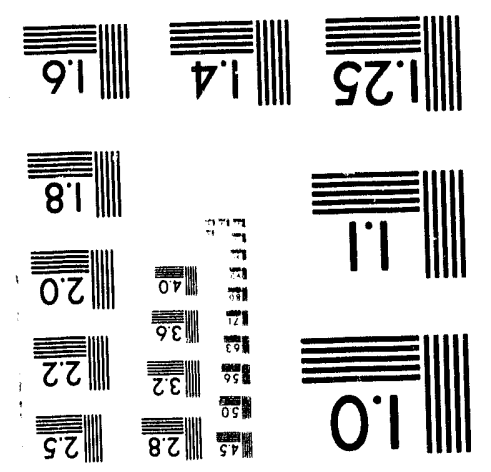



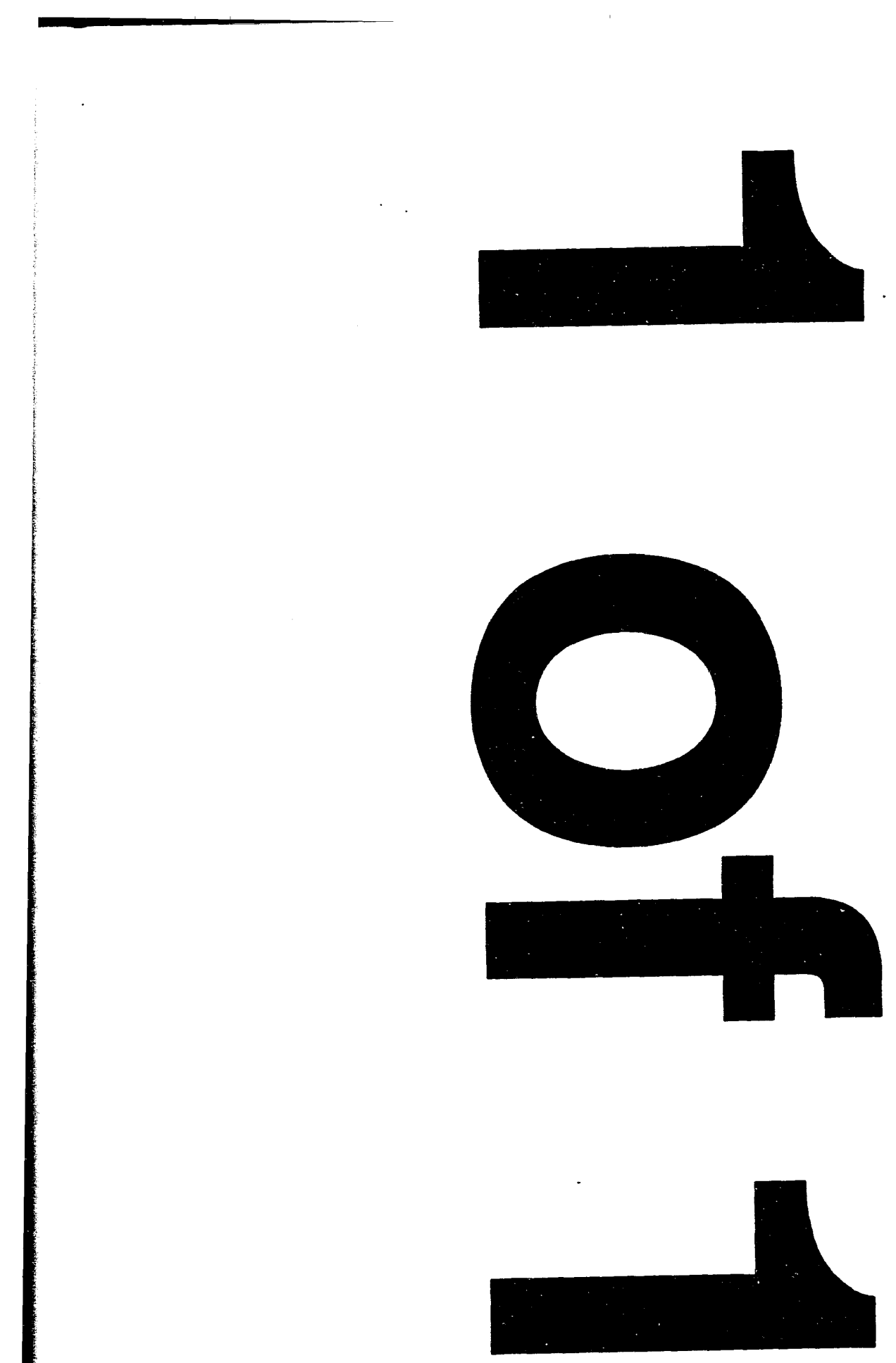


\title{
Observation of the Traveling Component of an X-Ray Standing Wave Field
}

\author{
B. Lai, W.B. Yun, J. Chrzas, and P.J. Viccaro \\ Advanced Photon Source, Argonne National Laboratory, 9700 S. Cass Ave., \\ Argonne, IL 60439
}

PACS no.: 42.10.Jd, 42.10.Qj, 78.65.-s, 78.70.Ck

\begin{abstract}
We present the first systematic experimental study of the traveling component of a standing wave field, formed by interference of an incident beam with its specularly reflected part from a plane interface. The traveling component of the standing wave field propagates parallel to the interface and its existence manifests the dynamical aspect of the standing wave field. In our experiment, a multilayer structure was used to aid in the observation of the traveling wave component. The data measured are explained using a finite thickness diffraction grating model.
\end{abstract}


Standing-wave-based $x$-ray techniques have been applied successfully in recent years to probe a broad range of material systems on their interfacial structures and elemental distribution. ${ }^{1}$ A standing wave field can be produced from the interference of two mutually coherent waves, and it exists in the region where the two interfering beams overlap. To create the standing wave field, an incident monochromatic $x$-ray beam may interfere with either the beam Bragg-reflected from a crystal, ${ }^{2}$ a layered synthetic microstructure $\mathrm{LSM}^{3}$ or with the beam totally reflected from a flat surface. ${ }^{4}$ The static intensity distribution of the standing wave field normal to the reflecting surface is of sinusoidal form, and its period, $p=\lambda / 2 \sin \theta$, can be varied by

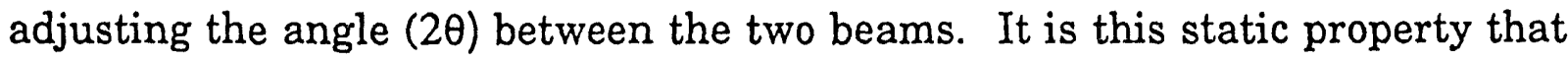
has been employed in all the standing-wave-based $\mathrm{x}$-ray techniques currently in use. However, the standing wave field also consists of a traveling component, which manifests the dynamical aspect of the standing wave field. To our knowledge, the existence of this traveling component and its application to structural analysis have not been investigated. We report in this work the first direct experimental demonstration of this traveling field. Theoretical calculations on diffraction of the traveling wave by a multilayer structure confirmed our experimental results.

Fig. 1a shows a schematic of the experimental setup. X-rays from a sealed $\mathrm{Cu}$ tube (2kW power) were monochromatized by a $\mathrm{Si}(111)$ crystal and collimated with two slits of $0.1 \mathrm{~mm}$ width placed before and after the crystal. $\mathrm{Cu} \mathrm{K}_{\alpha}$ radiation $(1.541 \AA$ ) was selected to incident on the sample at a glancing angle. The sample was mounted on the $\theta$ stage of a precision $\theta-2 \theta$ goniometer. On the arm of the $2 \theta$ stage, a NaI scintillation counter was mounted at $498 \mathrm{~mm}$ from the center of rotation to collect radiation scattered from the sample. A 
narrow vertical slit of $0.3 \mathrm{~mm}$ placed in front of the detector provided an angular definition of better than $0.035^{\circ}$.

The sample consisted of a $250 \AA$ thick Pt film sputtered onto a flat $\mathrm{Si}(001)$ substrate. Because the $\mathrm{x}$-ray refractive index of $\mathrm{Pt}$ film is less than unity, there exists a critical angle for total reflection $\alpha_{c}$. For incident angles less than $\alpha_{c}$, the incident $\mathrm{x}$-ray beam is nearly totally reflected. At the $\mathrm{Cu} \mathrm{K}_{\alpha}$ line, the critical angle is $0.57^{\circ}\left(\alpha_{c}^{\mathrm{Pt}}\right)$. The e-fold penetration depth for $\mathrm{x}$-rays in $\mathrm{Pt}$ is about $10 \AA$ below the critical angle, and increases to $54 \AA$ at the critical angle. ${ }^{5}$ The $250 \dot{\mathrm{A}} \mathrm{Pt}$ film is thus thick enough to reflect all incoming $\mathrm{x}$-rays in the total external reflection regime. The reflected beam interferes with the incident beam, and an electric field with total amplitude of $A\left(x, z, \vec{k}, \vec{k}^{\prime}\right)$ is established above the Pt surface. Using the $X-Z$ coordinate notation in Fig. $1 b$,

$$
\begin{aligned}
A\left(x, z, \vec{k}, \vec{k}^{\prime}\right) & =e^{i(\vec{k} \cdot \vec{r}-\omega t)}+r \cdot e^{i\left(\overrightarrow{k^{\prime}} \cdot \vec{r}-\omega t\right)} \\
& =2|r| \cos \left(k_{z} z+\phi / 2\right) e^{i\left(k_{x} x-\omega t+\phi / 2\right)}+(1-|r|) e^{i\left(k_{x} x-k_{z} z-\omega t\right)}
\end{aligned}
$$

where $r\left(=|r| e^{i \phi}\right)$ is the Fresnel reflection coefficient, $\vec{k}$ and $\vec{k}^{\prime}$ are the wavevectors of the incident and the reflected beam respectively, and $\omega$ is the angular frequency of the electric field. The phase shift $\phi$ is determined by the refractive indices of the two media involved and varies from $\pi$ to 0 as the glancing angle of incidence increases from $0^{\circ}$ to the critical angle $\alpha_{c} .^{4}$

The first term of Eq. 1 represents a traveling wave propagating parallel to the surface. Its existence unveils the dynamical nature of the interference field. The amplitude of this traveling wave, $2|\mathrm{r}| \cos \left(\mathrm{k}_{\mathrm{z}} \mathrm{z}+\phi / 2\right)$, varies as a function of the distance $z$ from the reflecting surface and the incident angle $\alpha_{0}$, as $\phi$ is a function of $\alpha_{0}$. The intensity distribution of the interference field is 
given by the absolute square of $\mathrm{A}\left(\mathrm{x}, \mathrm{z}, \overrightarrow{\mathrm{k}}, \overrightarrow{\mathrm{k}^{\prime}}\right)$, which yields the well-known static intensity distribution (standing wave field) normal to the surface. The second term in Eq. 1 represents a wave propagating in the direction of the incident wave with an amplitude of $1-|r|$. When the incident angle is less than the critical angle, this amplitude is small since $|r| \approx 1$ (absorption causes $|r|$ to be less than unity).

To observe the traveling component of the interference field, which exists in the region above the $\mathrm{Pt}$ surface, a $\mathrm{Si} / \mathrm{W}$ multilayer structure was deposited on top of the $\mathrm{Pt}$ film. In the presence of the multilayer, the wavefront of the traveling wave is modified due to different absorptions and phase delays in the multilayer's alternating material layers. The result is analogous to a diffraction grating of finite thickness with open and opaque zones that disturb the wavefront of an illumination wave, the traveling wave in our case. The traveling wave will be diffracted, and the diffraction intensity will depend on the strength of the interference field and the material contrast in the multilayer.

$\mathrm{Si} / \mathrm{W}$ multilayer has been shown to be efficient reflector in the hard $\mathrm{x}$-ray region. ${ }^{6}$ That results from the high contrast in their refractive indices: the imaginary part causes an absorption length of $3.1 \mu \mathrm{m}$ in $\mathrm{W}$ versus $70 \mu \mathrm{m}$ in $\mathrm{Si}$ for $\mathrm{Cu} \mathrm{K}_{\alpha}$ x-rays, while the real part induces a $\pi$-phase shift with respect to vacuum whenever the $\mathrm{x}$-ray travels $1.7 \mu \mathrm{m}$ in $\mathrm{W}$ versus $10.2 \mu \mathrm{m}$ in Si. In our experiment, $\mathrm{Si}$ and $\mathrm{W}$ layer pairs with $d$-spacing of $46.6 \dot{A}$ were deposited above the Pt film. The first order Bragg peak would occur at a $2 \theta$ angle of about $1.9^{\circ}$ for $8 \mathrm{keV} x$-rays. The periodicity was chosen to produce high quality $\mathrm{Si} / \mathrm{W}$ multilayer with the considerations described 
below. A sufficient number of layer pairs was deposited to enhance the scattered intensity of the traveling wave and the sharpness of the diffraction peaks, but not so many that the $x$-ray intensity reaching the Pt surface underneath would be greatly attenuated. Ten pairs were optimal for our conditions. To further reduce absorption, the thickness of the W layer was made to be $1 / 4$ of the $\mathrm{Si}$ layer. The low $\mathrm{W}$ ratio also serves to restrict the overall multilayer critical angle $\left(\alpha_{c}^{\mathrm{ml}}\right)$ to be less than that of the $\mathrm{Pt}\left(\alpha_{\mathrm{c}}^{\mathrm{ml}}<\alpha_{\mathrm{c}}^{\mathrm{Pt}}\right)$. This provides a large angular range of $\alpha_{0}$ in which the incident $x$-rays can penetrate into the multilayer and be totally reflected by the $\mathrm{Pt}$ film when $\alpha_{\mathrm{c}}^{\mathrm{ml}}<\alpha_{0}<\alpha_{\mathrm{c}}^{\mathrm{Pt}}$. A null sample was also prepared with the same $\mathrm{Si} / \mathrm{W}$ multilayer but without the $\mathrm{Pt}$ film. No standing traveling waves would be formed within the multilayer of the null sample because the critical angle of $\mathrm{Si}$ is smaller than $\alpha_{c}^{\mathrm{ml}}$. Both samples were prepared in a sputtering system with 8 " targets.

Fig. 2 shows the reflectivity $(\theta-2 \theta)$ curves measured on the two samples. The critical angle at $0.32^{\circ}$ for both curves was due to the $\mathrm{Si} / \mathrm{W}$ multilayer $\left(\alpha_{c}^{\mathrm{ml}}\right)$, while the one at $0.57^{\circ}$ for the solid line was caused by the $\mathrm{Pt}$ film $\left(\alpha_{c}^{\mathrm{Pt}}\right)$. In the region between the two critical angles, intensity modulation that continued into higher angles results from interference between the $x$-rays reflected from the air/multilayer interface and from the multilayer/Pt interface. ${ }^{7}$ The quality of the multilayer is apparent from the high reflectivity measured on the first order Bragg peak at $\alpha_{0}=1^{\circ}$. On the null sample, the Bragg peak appears at the same angle (identical $d$-spacing) but there is no critical angle due to $\mathrm{Pt}$. 
Diffraction of the traveling component of the standing wave field should be most discernible for incident angle $\alpha_{0}$ between $0.32^{\circ}$ and $0.57^{\circ}$. Spectra of $2 \theta\left(=\alpha+\alpha_{0}\right)$ scan taken at several fixed incident angles $\alpha_{0}$ within that range are shown in Fig. 3. During measurement, the samples were held at a constant angle to the incident $x$-ray while the detector was scanned to map out the angular intensity of the scattering. A peak at about $2^{\circ}$ was found in all the spectra. Its shift toward higher angles at small $\alpha_{0}$ was simply due to increased refraction correction. ${ }^{8}$ The peak is caused by the multilayers providing a fixed momentum transfer of $2 \pi / d$ to the incident beam. In other words:

$$
\Delta \mathrm{k}=2 \pi / \mathrm{d} \quad \rightarrow \quad \sin \alpha+\sin \alpha_{0}=\lambda / \mathrm{d} \quad,
$$

which means at small angles the angle of deflection $\alpha+\alpha_{O}$ is a constant $\lambda / \mathrm{d}=1.90^{\circ}\left(2.02^{\circ}\right.$ with refraction). Since it is due to momentum transfer provided by the multilayer, the peak is found on both samples. These nonspecular scattering can be used to measure the $d$-spacing, and in fact have been applied previously for that purpose. ${ }^{7,9}$

Besides the first peak, an additional peak (indicated by arrows) was observed at higher angles only on the sample containing the $\mathrm{Pt}$ film. This peak was clearly observable for a limited angular range, $\alpha_{c}^{\mathrm{ml}}<$ $\alpha_{0}<\alpha_{c}^{\mathrm{Pt}_{\mathrm{t}}}$. The fact that it nearly disappeared when the incident angle was greater than $\alpha_{c}^{\mathrm{Pt}}$ indicated it relied on the high reflectivity provided by the Pt. At the other limit, when the incident angle became less than $\alpha_{c}^{m l}, x$-rays could not penetrate through the multilayer and this peak was not observed. In between the two critical angles, it moved to smaller angles as $\alpha_{0}$ decreased. This peak can be accurately accounted for by considering diffraction of the traveling wave by the multilayer 
structure. A similar diffraction pattern has been experimentally observed in the past for Langmuir-Blodgett film on glass surface (doublet structure), ${ }^{9}$ but its origin remained unknown.

The traveling wave propagates inside the multilayer structure in the $x$-direction. The wavefront traveling in the $\mathrm{W}$ layer experiences stronger intensity attenuation and larger phase delay compared to those that travel in the adjacent $\mathrm{Si}$ layers. Distortion in the wavefront increases as the traveling wave propagates in the multilayer, and that results in diffraction signals observed at angle $\alpha$. The diffracted intensity $I(\alpha)$ can be calculated from the Fraunhofer integral ${ }^{10}$ :

$$
\mathrm{I}(\alpha) \sim\left|\int_{0}^{10 \alpha} \mathrm{A}(\mathrm{z}) \mathrm{T}(\mathrm{z}) \mathrm{e}^{\mathrm{ikz} \sin \alpha} \mathrm{dz}\right|^{2}
$$

where $A(z)=e^{i\left(n k_{z} z\right)}+r \cdot e^{-i\left(n k_{z} z\right)}$ is the complex amplitude of the interference fields (see Eq. 1) with $\mathrm{n}$ being the average complex refractive index of the $\mathrm{Si} / \mathrm{W}$ multilayer, and $\mathrm{T}(\mathrm{z})$ is the transmission function of the multilayer. $T(z)$ is periodic in $z$ with period $d$ and thus can be expanded in a Fourier series $\Sigma_{m} t_{m} e^{i(2 \pi m / d) z}, m=$ integer. Then the total phase factor in the Fraunhofer integral becomes $\left( \pm n k_{z} z+2 \pi m z / d+k z \sin \alpha\right)$, which is stationary in $z$ only when:

$$
\sin \alpha=\mathrm{m} \lambda / \mathrm{d} \pm \mathrm{nkz} / \mathrm{k}=\mathrm{m} \lambda / \mathrm{d} \pm \mathrm{n} \sin \alpha_{0} .
$$

In other words, for each of the $\mathrm{m}^{\text {th }}$ order, the diffraction peaks are divided with a splitting of $2 n \sin \alpha_{0}$. For small angles, the splitting decreases linearly with the incident angle, which is in agreement with what we have observed from experimental data (see Fig. 3). If the standing wave period $\left(\lambda / \sin \alpha_{0}\right)$ is smaller than the $d$-spacing, the splitting is large enough that peaks from different orders will overlap. 
The period of the standing wave field for our sample is $155 \dot{\mathrm{A}}$ at $\alpha_{\mathrm{c}}^{\mathrm{pt}}$, and therefore no overlaps of orders will occur. Note for the null sample $r=0$, and the condition for angular maxima of Eq. 4 reduces to Eq. 2.

The calculated diffraction intensity based on Eq. 3 for a 10-period multilayer is showed in Fig. 4, after correcting $\alpha$ and $\alpha_{0}$ for refraction at the air-multilayer interface. In the calculation, we assumed the $x$-ray transmission of $S i$ and $W$ are $T(z)=1$ for $S i$ layers and $T(z)=0$ for $W$ layers. At the first order $(m=1)$, there is splitting in the diffraction peak (A and B) as predicted, and the calculated peak positions agree very well with the experimental results. The difference in the measured peak heights of A and B (Fig. 4) results from the asymmetric amplitude distribution of the interference field $A(z)$, due to nonideal reflection from $\mathrm{Pt}(|\mathrm{r}|<1)$ and absorption of the incident and reflected beam in the multilayer. In our calculation of the amplitude of the incident and reflected beams, the actual optical constants of $\mathrm{Si}, \mathrm{W}$, and $\mathrm{Pt}$ were used to reflect the asymmetric distribution in $A(z)$. Overall, the calculated result agrees very well with measured data. For three different incident angles, Eq. 3 predicted correctly the absolute angular locations of all peaks in the data. Note that the calculated peak intensities yield reasonable agreement with the measured data. The residual difference could be due to instrumental broadening, lower material contrast and finite length of travel in the multilayer, and dynamical corrections, which will be studied in later experiments.

The length scale for scattering of the traveling wave in the multilayer can be estimated. In reciprocal space, lattice points 
representing the multilayer are separated vertically by $1 / d$. In order for a horizontal wavevector to be scattered by an angle $\alpha$ to the $m=1$ order, the reciprocal lattice points must have a horizontal width of at least $\approx \alpha / d$ for small $\alpha$. In real space, that means the average scattering length in the multilayer is no more than $d / \alpha=5400 \dot{A}$ for $\alpha \sim 2^{\circ}$. This is reasonable considering the phase-shifting capability of the $W$ and that the differential absorption between $\mathrm{Si}$ and $\mathrm{W}$ also contributes to the scattering.

In conclusion, we have demonstrated both experimentally and theoretically the existence of a traveling component in a standing wave field, formed by a collimated monochromatic incident $x$-ray beam and its reflected part from a flat interface. The traveling wave propagates parallel to the reflection surface, and its amplitude along the surface normal is modulated. Spatial variation of optical constant in the interference field results in diffraction of the traveling wave, and this phenomenon can be applied to structural determination of surfaces and interfaces.

Acknowledgement: This work was supported by the U.S. Department of Energy, BES-Materials Science, under contract W-31-109-Eng-38. 


\section{References}

1. M. J. Bedzyk, G. Materlik, and M. V. Kovalchuk, Phys. Rev. B 30, 2453 (1984); K. Akimoto, T. Ishikawa, T. Takahashi, and S. Kikuta, Jpn. J. Appl. Phys. 22, L798 (1983); J. A. Golovchenko, J. R. Patel, D. R. Kaplan, P. L. Cowan, and M. J. Bedzyk, Phys. Rev. Lett. 49, 560 (1982).

2. P. L. Cowan, J. A. Golovchenko, and M. F. Robbins, Phys. Rev. Lett. 44, 1680 (1980).

3. T. W. Barbee and W. K. Warburton, Materials Lett. 3, 17 (1984); M. J. Bedzyk, D. H. Bilderback, G. M. Bommarito, M. Caffrey, and J. S. Schildkraut, Science 241, 1788 (1988).

4. M. J. Bedzyk, G. M. Bommarito, and J. S. Schildkraut, Phys. Rev. Lett. 62, 1376 (1989).

5. W. B. Yun and J. M. Bloch, J. Appl. Phys. 68, 1421 (1990).

6. T. Ishikawa, A. Iida, and T. Matsushita, Nucl. Instr. and Methods A246, 348 (1986).

7. J. Sauro, I. Fankuchen, and N. Wainfan, Phys. Rev. 132, 1544 (1963).

8. R. W. James, The Optical Principles of the Diffraction of X-Rays (Ox Bow, Woodbridge, Connecticut, 1982).

9. D. S. Kapp and N. Wainfan, Phys. Rev. 138, A1490 (1965).

10. M. Born and E. Wolf, Principles of Optics, $6^{\text {th }}$ ed. (Pergamon Press, New York, 1980). 


\section{Figure Captions}

Fig. 1. Schematic of (a) experimental setup, (b) the sample, and the notation used in the text.

Fig. 2. Reflectivity $(\theta-2 \theta)$ scans on sample with (solid line) and without (dotted line) the Pt layer.

Fig. 3. Scattered intensity as a function of the deflection angle. Solid lines: sample with $\mathrm{Pt}$ layer. Dots: null sample. The intensities have been normalized.

Fig. 4. Histogram: experimental data. Solid line: modelling using Eq. 3 for $m=1$. 


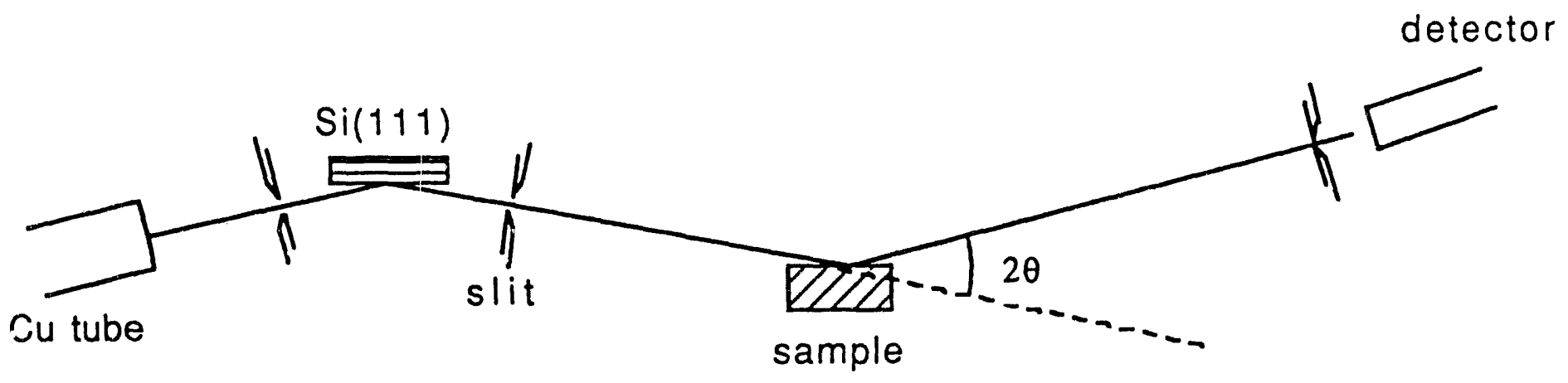

Fig. Ia 


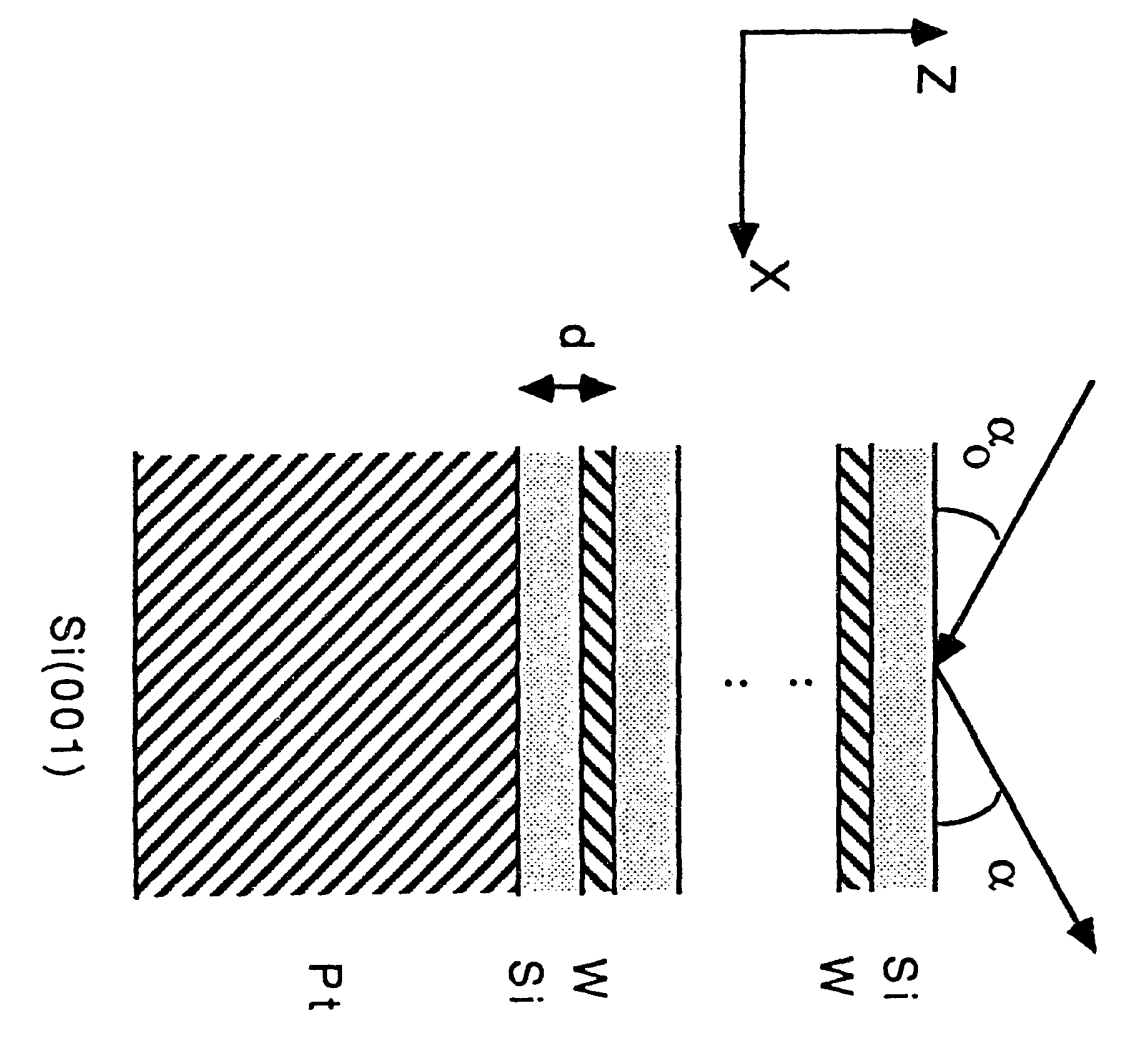

$\frac{\pi}{0}+$ 
Intensity

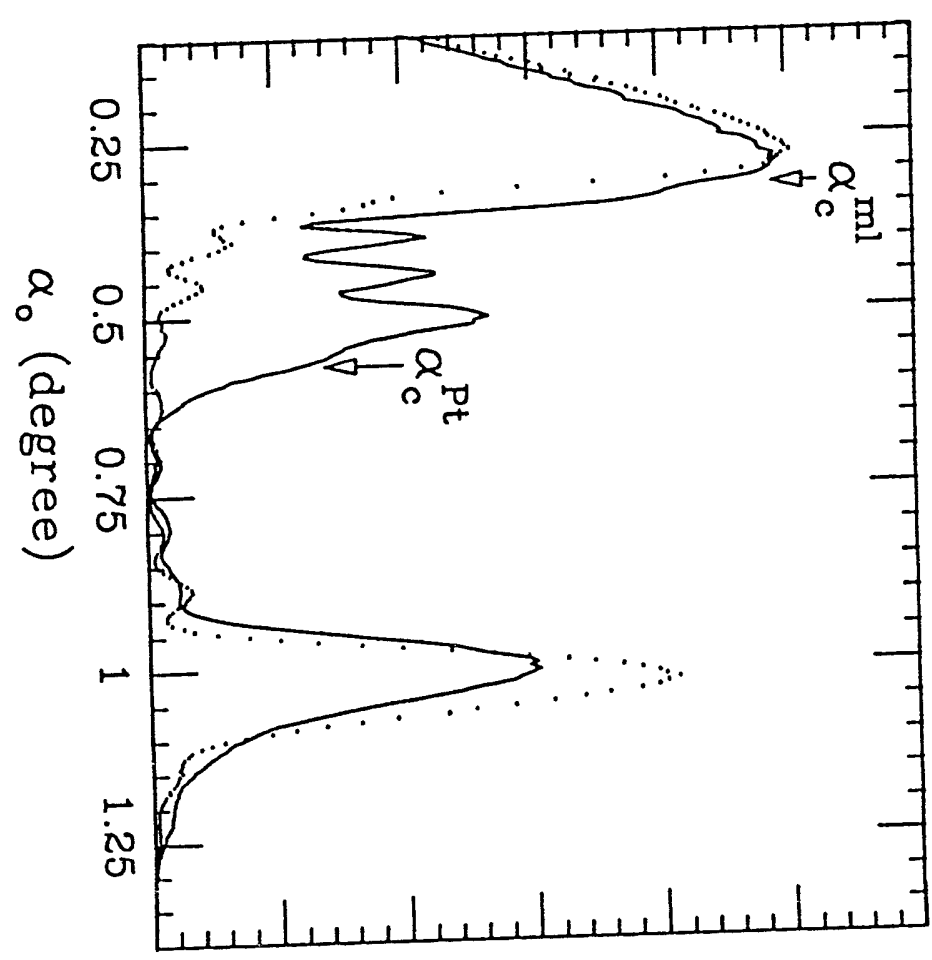

$\pi$
$N$ 


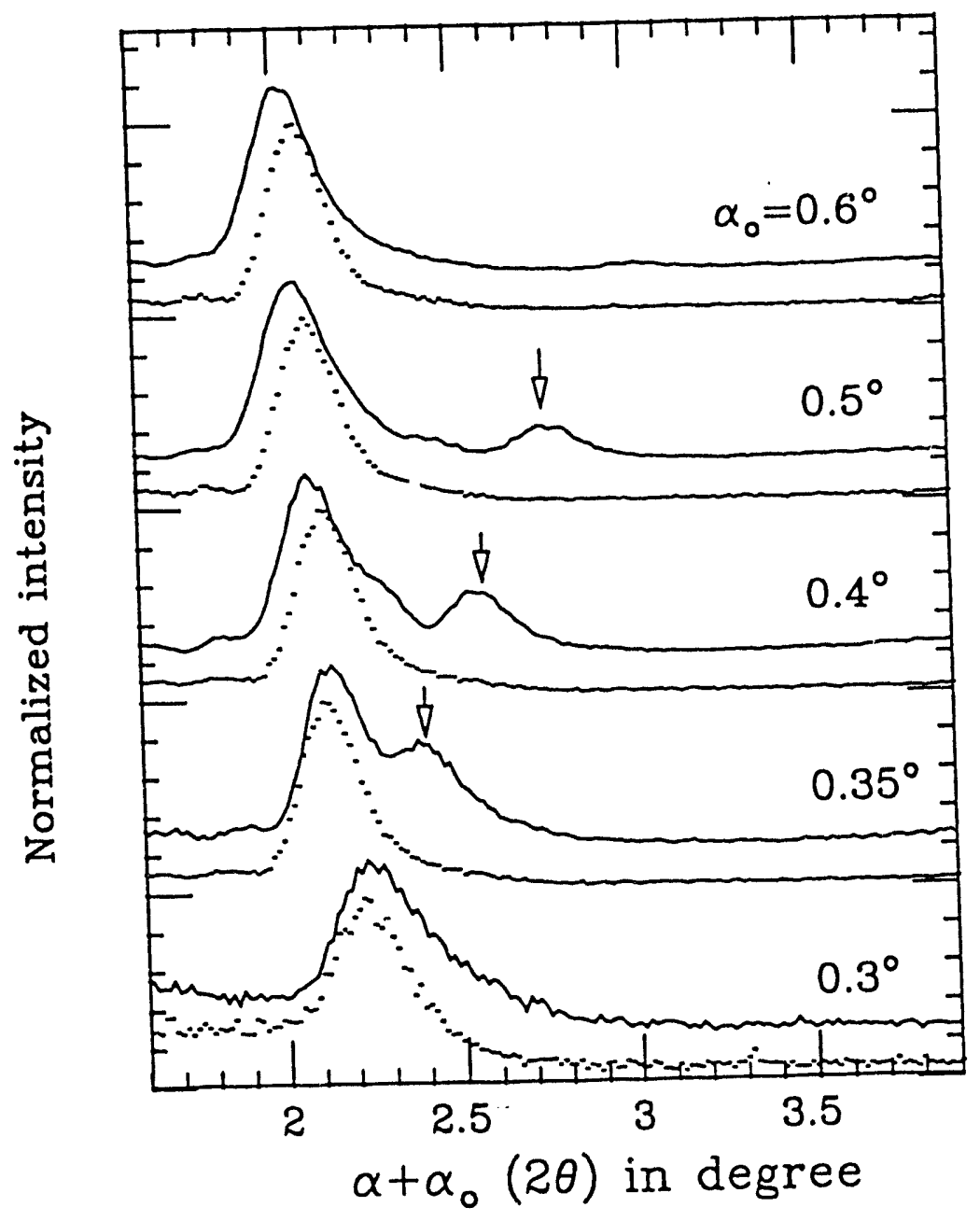

Fig. 3 


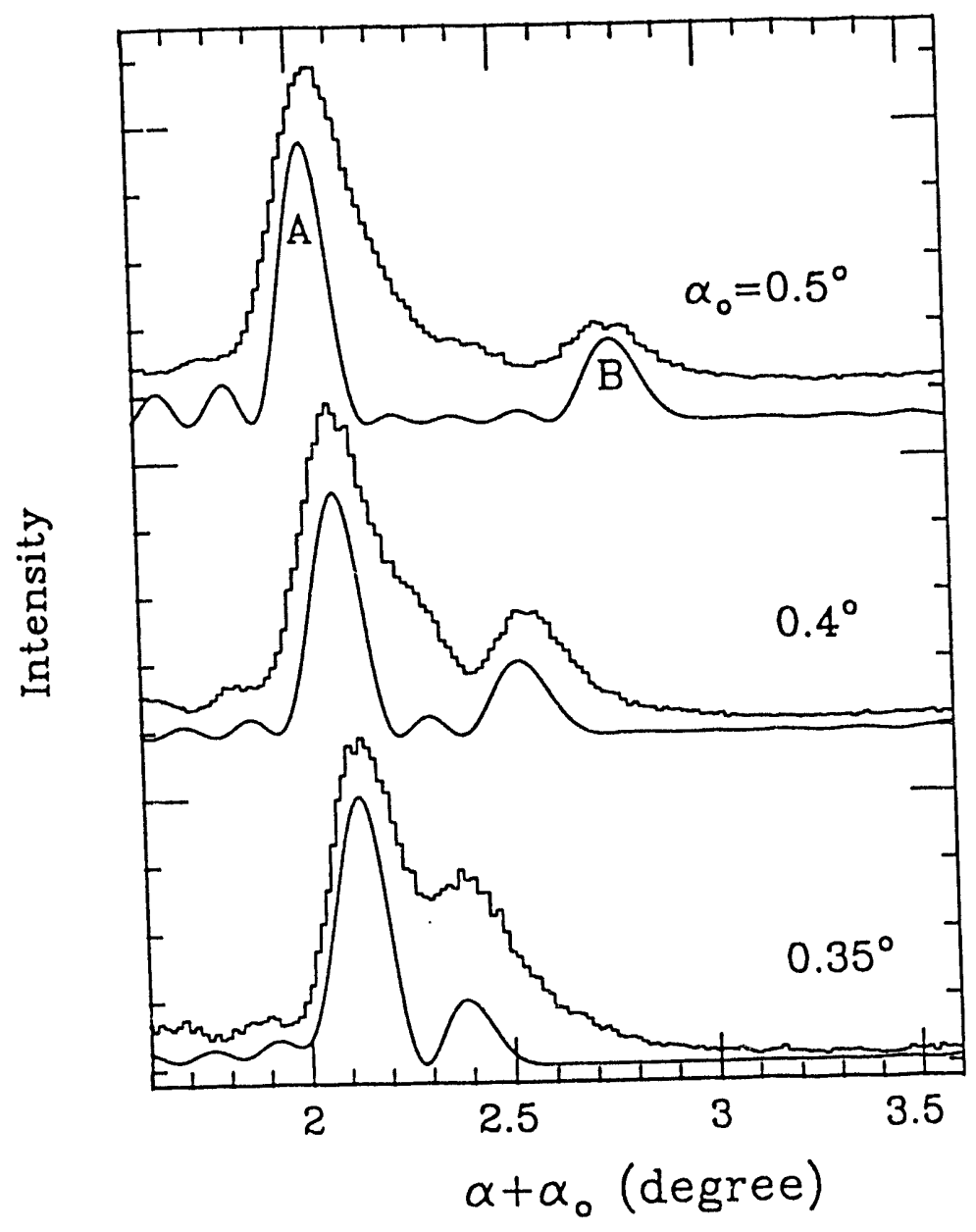

Fig: 4 

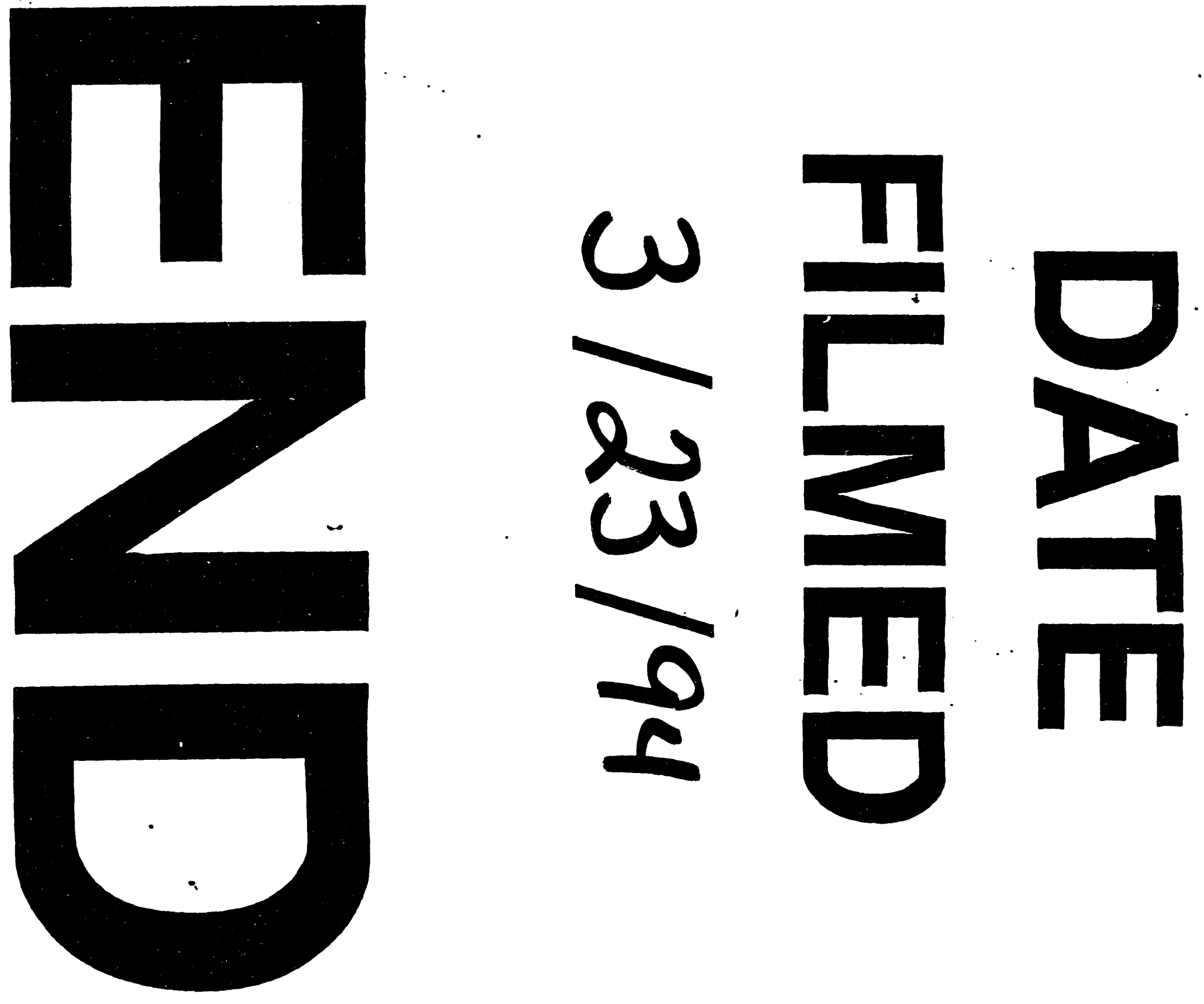
